\title{
Finite element analysis of an eddy current heater for wind or water kinetic energy conversion into heat
}

\author{
O. Nebi, V. Firețeanu \\ EPM_NM Laboratory, http://amotion.pub.ro/ epm/ \\ POLITEHNICA University of Bucharest, 313 Splaiul Independenței, 060042 Bucharest, Romania \\ E-mails: onur.nebi@amotion.pub.ro, virgiliu.fireteanu@upb.ro
}

\begin{abstract}
The paper presents 2D and 3D finite element models for the analysis of the electromagnetic and thermal fields and the local and global quantities in an eddy current heater with permanent magnets, able to convert the wind or water kinetic energy into heat.

Correlations between the input and output operational parameters of the heater are studied taking into account the temperature dependence of physical properties and the magnetic nonlinearity.
\end{abstract}

\section{Key words}

Eddy currents heater, finite element analysis.

\section{Introduction}

The humanity becomes more and more interested in producing electrical energy from renewable sources based on wind or water motion. An important part of the electrical energy produced worldwide is converted into heat. In this context, this paper studies a device able to convert into heat the kinetic energy of wind or water trough Joule effect of eddy currents [1] - [4].

\section{Eddy current heater description}

The finite element analysis concerns the heating system in Fig. 1, able to convert into heat the kinetic energy of the rotor motion.

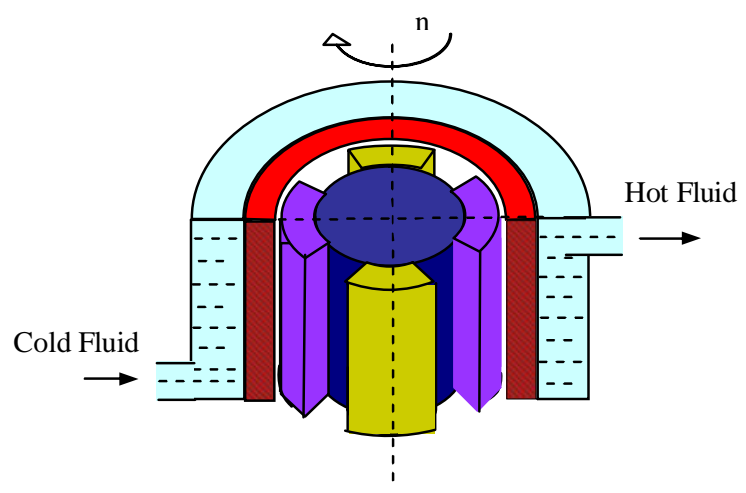

Fig. 1. Eddy current heater principle
The device contains a rotor assembly, consisting in a magnetic core surrounded by permanent magnets, and a tube type stator, witch represents the heating element.

The stator is surrounded by an annular chamber, in which the fluid to be heated flows. This heater is coupled to a wind or water turbine. The heat generated in the stator is the result of the Joule effect of eddy currents as result of rotor motion. This heat is transferred outside the heater through a liquid or a gas flow.

\section{The $2 D$ finite element analysis of the eddy current heater 1}

This chapter analyzes the electromagnetic and thermal fields of the heater 1 in Fig. 2, respectively the eddy currents, the induced power and the temperature field in this device based on $2 \mathrm{D}$ plan models. The electromagnetic computation domain includes the following regions:

- ROTOR, a non-conducting and magnetic nonlinear volume region, whose magnetization is defined by the saturation $2 \mathrm{~T}$ and the initial relative permeability 2000;

- MAG_N, MAG_S, permanent magnet volume regions with positive, respective negative radial magnetization, characterized by the remanent flux density $1 \mathrm{~T}$ and relative permeability 1.1 ;

- A_G and AIR, air volume regions between the rotor and stator;

- STATOR, a conducting and magnetic nonlinear volume region with saturation $1.9 \mathrm{~T}$, initial relative permeability 2000 and linear temperature $\theta$ dependent resistivity, expressed by the formula $0.5 \cdot 10^{-6}\left[1+1.5 \cdot 10^{-3} \cdot(\theta-20)\right] \Omega \mathrm{m}$;

- STATOR_EXT, the surface region that is the computation domain boundary, where the magnetic field has tangential orientation.

The thermal computation domain includes the STATOR region, whose material is characterized by the temperature dependent thermal conductivity $54 \cdot\left[1-3.846 \cdot 10^{-4} \cdot(\theta-20)\right] \mathrm{W} / \mathrm{m} /{ }^{\circ} \mathrm{C}$ and specific heat $7800 \cdot 450 \cdot\left[1+4.545 \cdot 10^{-4} \cdot(\theta-20)\right] \mathrm{J} / \mathrm{m}^{3} /{ }^{\circ} \mathrm{C}$, and the surface boundary regions STATOR_INT and STATOR_EXT, characterized by convection heat transfer.

The inner diameter of stator is $160 \mathrm{~mm}$ and the global quantities correspond to $300 \mathrm{~mm}$ heater axial dimension. 


\section{A. Analysis of heater electromagnetic parameters}

The step by step in time domain finite element computation of the transient electromagnetic field considers the value $\Delta \alpha=1.44$ degrees of the angular step. For the value $n=1000 \mathrm{rpm}$ of rotor speed, the corresponding time step is $\Delta \mathrm{t}=60 \Delta \alpha /(2 \pi \mathrm{n})=0.24 \mathrm{~ms}$. The results presented in this section correspond to the steady state device operation, when the induced power no more depends on time variable.

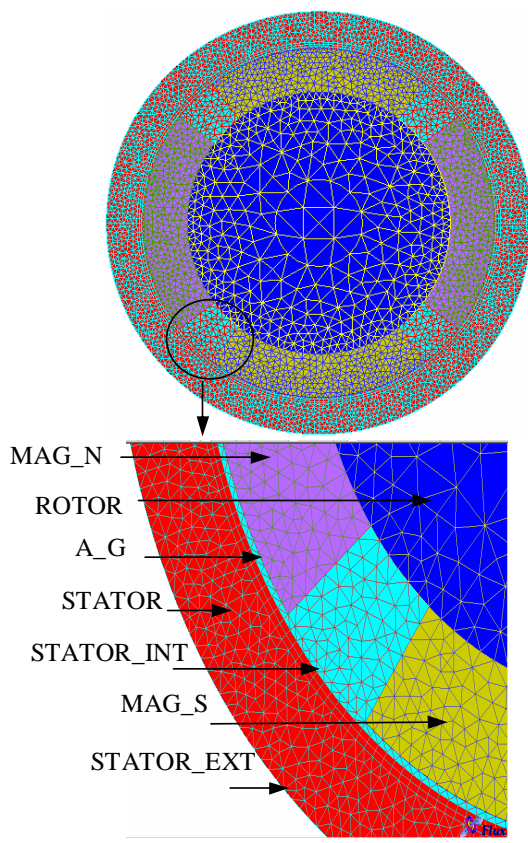

Fig. 2. Geometry of the electromagnetic computation domain

The chart of the magnetic flux density in the entire computation domain is presented in Fig. 3 for a device with four permanent magnets/poles.

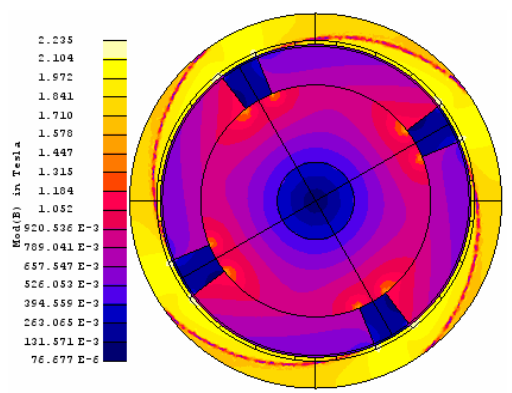

Fig. 3. The chart of the magnetic flux density

Different numbers of poles where considered in order to see the influence of this parameter on the induced power for imposed value of rotor speed. As presented in Fig. 4, the maximum of induced power for the speed $1000 \mathrm{rpm}$ corresponds to the rotor with four poles.

The charts of the induced current density, Fig. 5, emphasize the decrease of the electromagnetic field penetration in the stator when the number of rotor poles increases. In connection with this result, the influence of the stator thickness on the induced power was studied for the four poles heater and the speed $1000 \mathrm{rpm}$. Fig. 6 shows that the induced power does not more increase for values of the stator thickness over $16 \mathrm{~mm}$.

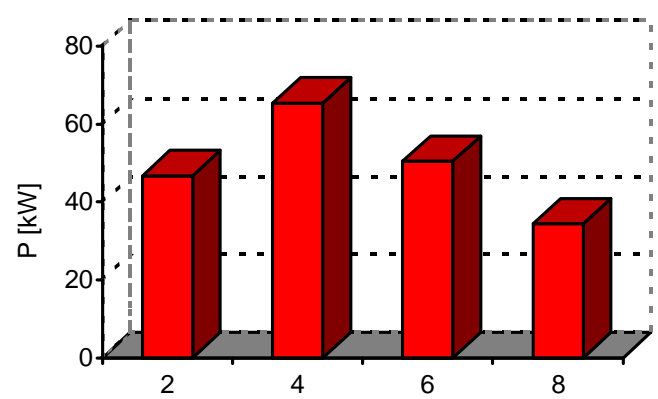

Fig. 4. Dependence of induced power on number of poles

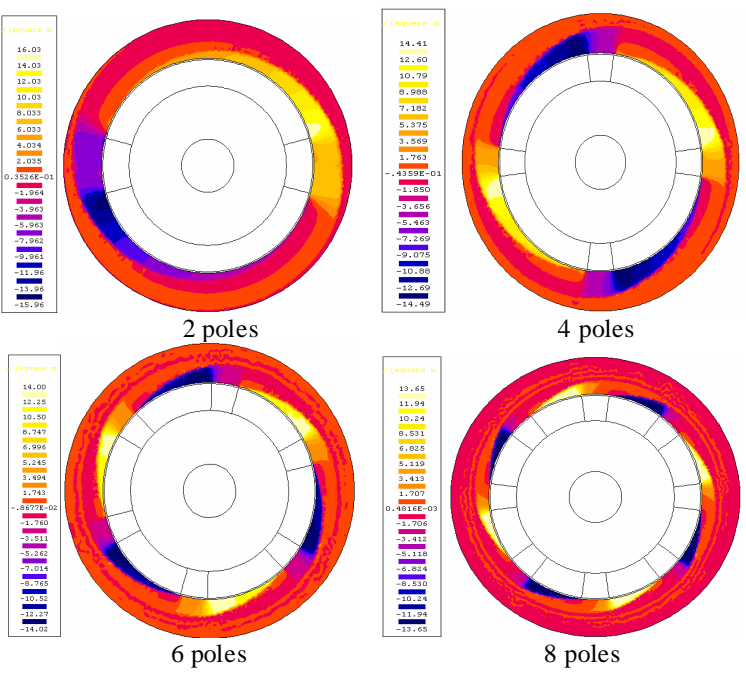

Fig. 5. Charts of induced current density for different number of poles

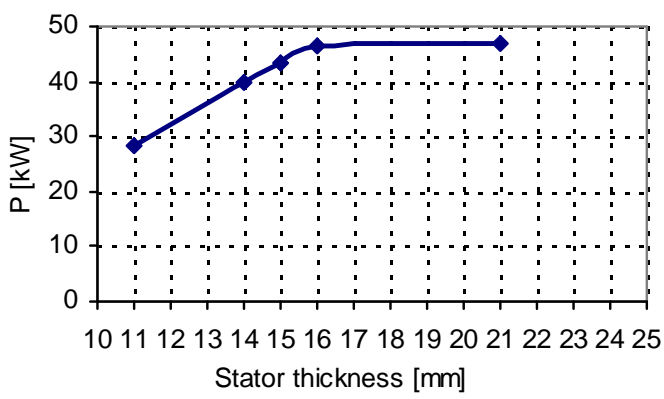

Fig. 6. Dependence of induced power on stator thickness

\section{B. Evaluation of heater characteristics based on the transient magnetic - transient thermal link}

The main interest for the eddy current heater operation concerns the steady state values of induced power and of temperature field of the stator armature. These results are evaluated in this section through the link between the analysis of the transient electromagnetic field followed 
by the transient analysis of the stator heating as effect of the steady state Joule power of eddy currents. Such a link supposes two successive steps. First step concerns a transient magnetic analysis for the values of the electromagnetic properties at ambient temperature until electromagnetic steady state is reached. For this state is computed the mean values in time of the local induced power density in the stator over a period $\mathrm{T}$ of time variation of this quantity. For the four poles heater and the speed $1000 \mathrm{rpm}$ the electromagnetic period of the local induced power density is $\mathrm{T}=15.12 \mathrm{~ms}$. Fig. 7 shows the time variation of the instantaneous power density in a point on the inner stator surface.

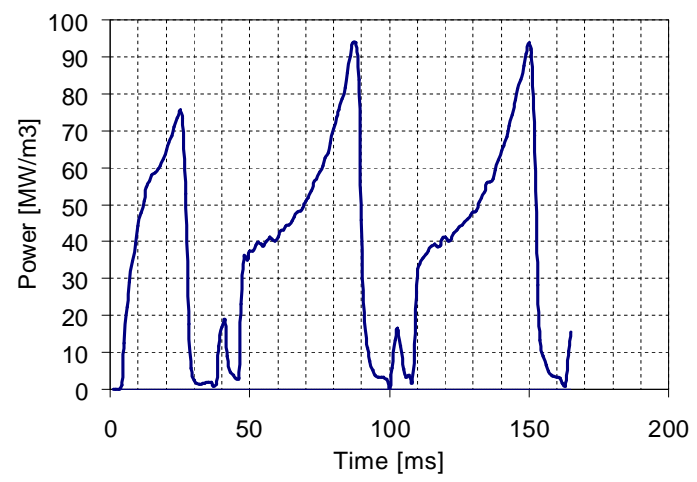

Fig. 7. Time variation of the induced power volume density in a point during electromagnetic transient

Charts of the volume density of the instantaneous induced power density in stator for four time steps during a period $\mathrm{T}$ of time dependence of this quantity are presented in the Fig. 8.

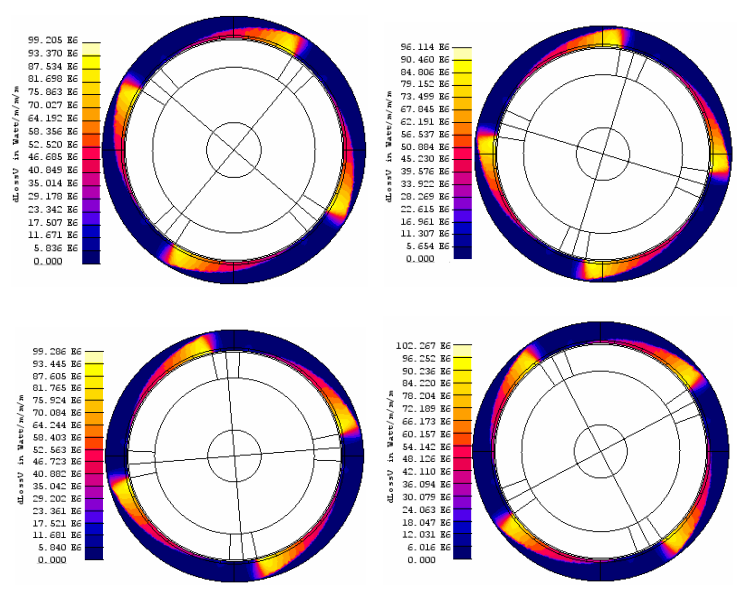

Fig. 8. Charts of instantaneous volume density of induced power

The chart of the mean value in time of the power density over a period $\mathrm{T}$ at the end of the electromagnetic transient, Fig. 9, shows the decrease in radial direction of the heating source that is this power density from the inner surface of the stator to the outer surface, where this source vanishes. This field of the power density is the source of the transient thermal problem, which represents the second step in the evaluation of the heater parameters. As Fig. 10 shows, the electromagnetic transient is practically finished after $15 \mathrm{~ms}$. The steady state value of the induced power, respectively the power of the heater corresponding to the rotor speed $1000 \mathrm{rpm}$ is $53 \mathrm{~kW}$.

The transient thermal analysis that offers the field of the stator temperature considers the convection thermal transfer to air on STATOR_INT surface region and to the water heated by the device on STATOR_EXT surface region. The time variation of the temperature in a point on the inner surface of the stator during the thermal transient is represented in Fig. 11 and the chart of the stator temperature at the end of thermal transient in Fig. 12.

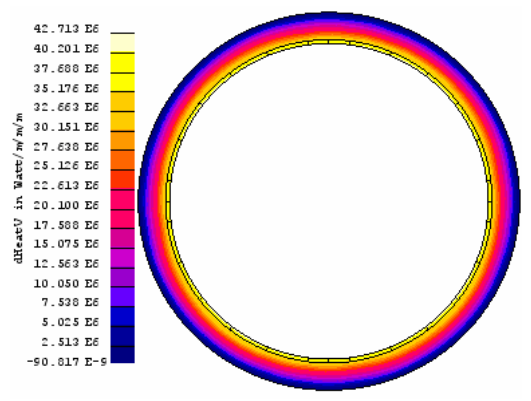

Fig. 9. Chart of the mean value in time of the instantaneous value of volume power density

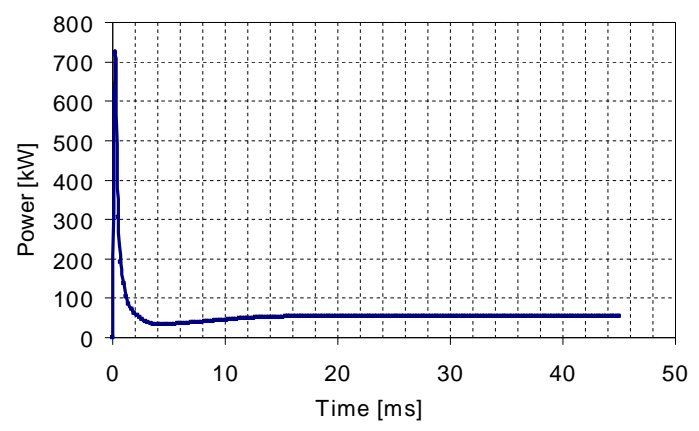

Fig. 10. Time variation of the induced power during electromagnetic transient

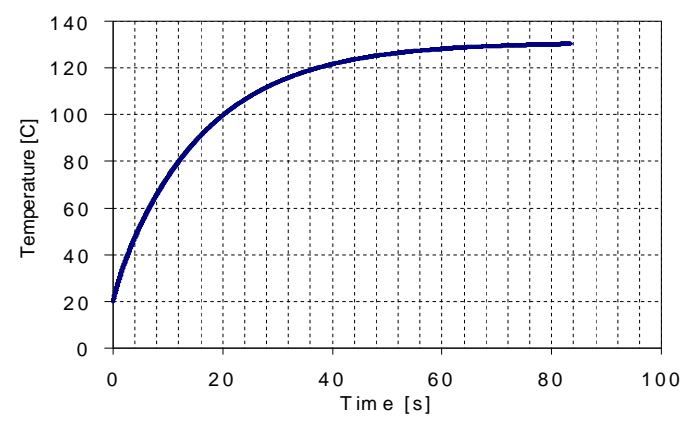

Fig. 11. Time variation of the temperature on the inner stator surface during thermal transient 


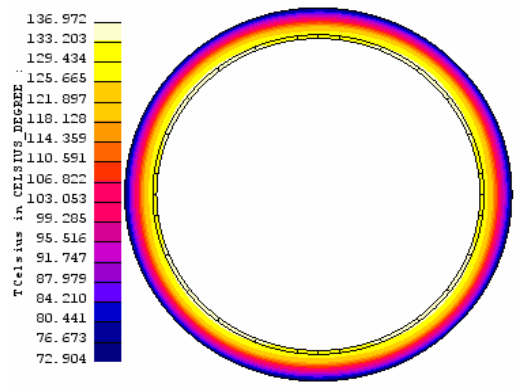

Fig. 12. The chart of the stator temperature at the end of the thermal transient

Taking into account the increase of the water temperature from the heater inlet to the outlet of $35^{\circ} \mathrm{C}$, this heater 1 is able to deliver $53000 /(1000 \cdot 4180) / 35=0.362 \cdot 10^{-3} \mathrm{~m}^{3} / \mathrm{s}=$ 21.7 litter of heated water per minute.

\section{Multiphysic coupling of magnetic and thermal transients}

Related to the step-by-step in time domain solution of the temperature in the previous section, the source of the heating offered by the magnetic computation is time independent. As consequence, this analysis is not able to take into account the temperature dependence of electromagnetic properties. In this section the step by step in time domain solutions of the magnetic and thermal problems are coupled in a particular manner imposed by the fact that the time step of the thermal problem is much higher than the magnetic time step.

Taking into account the ambient temperature as initial temperature field of the stator and the corresponding electromagnetic properties a first magnetic transient is performed until steady state is reached. Like previously, the mean values over the period $\mathrm{T}$ of the instantaneous volume density of induced power are computed. With this field of heating source, the temperature field corresponding to the first thermal time step is evaluated. The corresponding electromagnetic properties are then evaluated and the second magnetic transient is performed until steady state is reached. The mean value of the instantaneous induced power density is computed and then the second time step of temperature computation is performed. This multiphysic coupling offers the magnetic and thermal characteristics of the heater taking into account the temperature dependence of electromagnetic and thermal properties of the stator material. Each successive computation step includes a magnetic transient followed by the evaluation of the temperature field and the actualization of electromagnetic properties before passing to the next step.

If in the section $B$ the power induced in the stator has a constant value during the transient heating, now, with the multiphysic coupling that takes into account the increase of the stator resistivity when the temperature increases, the induced power, Fig. 13, has a slight decrease in time. The steady state value $44 \mathrm{~kW}$ of the induced power is under the result obtained in the section $3 B$.

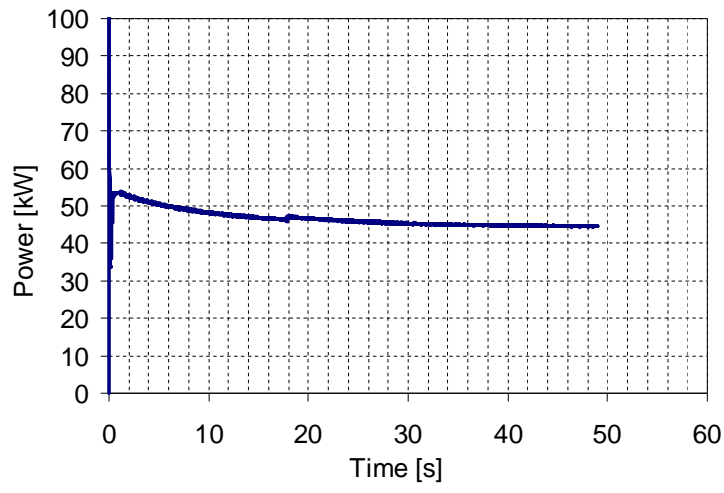

Fig. 13. Time variation of the induced power during the stator transient heating

The time variation of the temperature during heating transient in a point on the inner surface of the stator in case of the multiphysic coupling, Fig. 14, shows a steady state value of $112{ }^{\circ} \mathrm{C}$, under the similar result $130{ }^{\circ} \mathrm{C}$ of the section $3 B$.

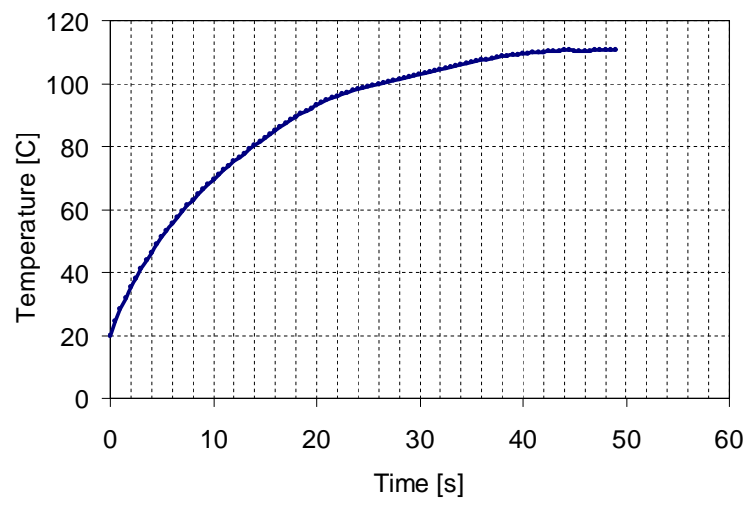

Fig. 14. Time variation of the temperature in a point on inner stator surface during transient thermal

The charts of the volume density of induced power and of the stator temperature for three different time steps during transient heating $\mathrm{t}_{1}=10 \mathrm{~s}, \mathrm{t}_{2}=49 \mathrm{~s}$ are presented in Fig. 15.
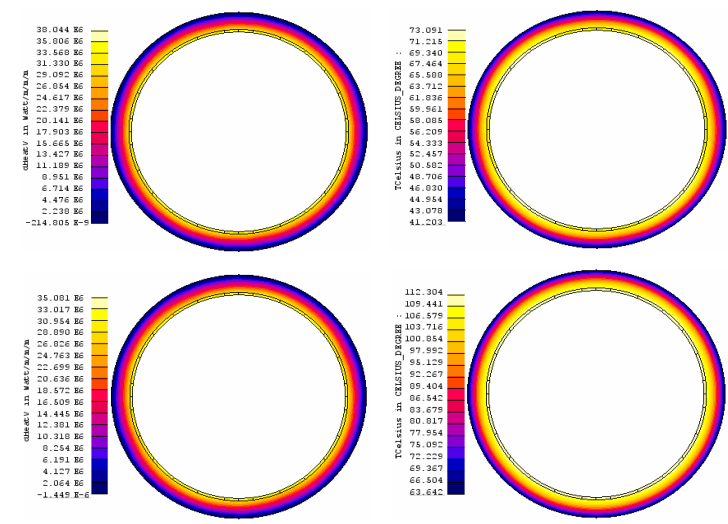

Fig. 15. Charts of the volume density of the induced power and the corresponding temperature 


\section{The 3D finite element analysis of the eddy current heater 2}

\section{A. Geometry and physical properties}

The eddy current heater 2 with four poles/permanent magnets studied in this chapter, Fig. 16, has a doubletube stator consisting in an aluminum tube inside another tube, magnetic steel made. Both tubes are eddy current regions, the outer operating also as stator magnetic armature.

The inner diameter of the stator is $162 \mathrm{~mm}$, the thickness of the aluminum tube is $10 \mathrm{~mm}$ and of the steel tube is $10 \mathrm{~mm}$. The axial dimension of the rotor is $40 \mathrm{~mm}$ and of the stator $60 \mathrm{~mm}$.

The aluminum tube has the temperature dependence of resistivity expressed by the formula $2.7 \cdot 10^{-8}\left[1+4.35 \cdot 10^{-3}(\theta-20)\right] \Omega \mathrm{m}$, the temperature dependence of the thermal conductivity $243\left[1-2.88 \cdot 10^{-4}(\theta-20)\right] \mathrm{W} / \mathrm{m} /{ }^{\circ} \mathrm{C}$ and of the specific heat $2700 \cdot 879\left[1+6.71 \cdot 10^{-4}(\theta-20)\right] \mathrm{J} / \mathrm{m}^{3} /{ }^{\circ} \mathrm{C}$. The steel tube has the magnetic relative permeability 500 , the resistivity $0.25 \cdot 10^{-6} \Omega \mathrm{m}$, the thermal conductivity $54 \mathrm{~W} / \mathrm{m} /{ }^{\circ} \mathrm{C}$ and the specific heat $7800 \cdot 450 \mathrm{~J} / \mathrm{m}^{3} /{ }^{\circ} \mathrm{C}$.

The rotor magnetic core is non-conductive steel made with relative permeability 2000 . The magnets properties are those defined in Chapter 3.

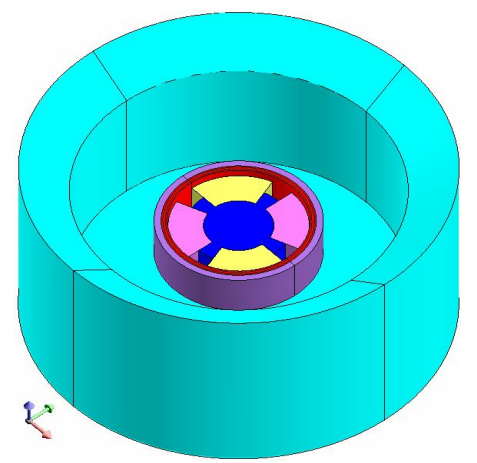

Fig. 16. The 3D geometry of the double-tube stator eddy current heater 2

Taking into account the symmetries of the device, the computation domain of the electromagnetic field, Fig. 17, represents only $1 / 8$ of the device in Fig. 16. This 3D computation domain includes two mechanical sets, a mobile one of the rotor and the second fix of the stator, separated by a compressible region that is the air gap between the stator and rotor armatures.
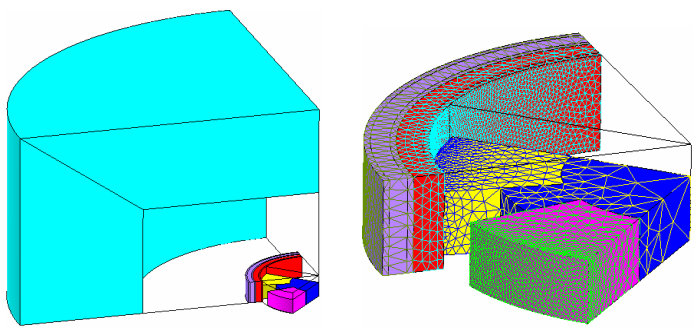

Fig. 17. Computation domain of the electromagnetic field
The computation domain of the thermal field, Fig. 18, considers two volume regions WP and RING of thermal conduction type and the surface regions AIR, WATER, of convection thermal transfer type.

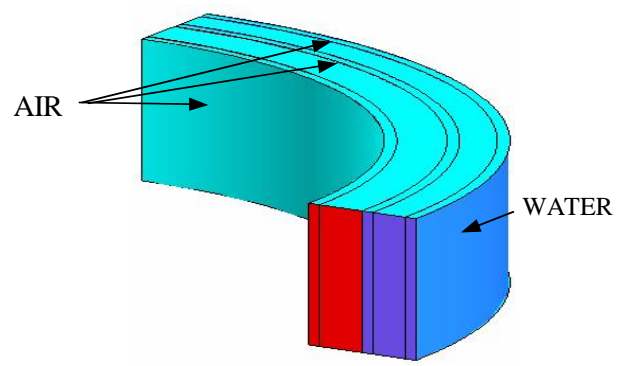

Fig. 18. Computation domain of the thermal field

\section{B. New multiphysic solution of the 3D magnetic and thermal transients}

The finite element model of the heater 2 analyses the transient 3D electromagnetic field and the transient heating of the stator as result of Joule effect of the induced currents. A step-by-step type in time domain solving process is considered. The rotor position changes each time step $\Delta \mathrm{t}$, corresponding to the elementary angle $\Delta \alpha=(2 \pi \mathrm{n} / 60) \Delta \mathrm{t}$, where $n$ is the rotor speed rpm. Taking into consideration a small value of this angle in order to have a good approximation of real electromagnetic transient, a very large time of the solving process it results.

As seen in Chapter 3, the heating transient is characterized by a time constant much higher than the time constant of electromagnetic transient. In order to realize the multiphysic coupling of the electromagnetic and thermal transients with the same value of the time step for the two problems, hypothetic values of the specific heat are considered. All hypothetic materials of the thermal computation domain have a specific heat $\mathrm{k}$ time lower than the real value. The differential equation:

$$
\gamma c \frac{\partial \theta}{\partial t}=\left(\frac{\gamma c}{k}\right) \frac{\partial \theta}{\partial(t / k)}=\left(\frac{\gamma c}{k}\right) \frac{\partial \theta}{\partial\left(t_{e}\right)}=\operatorname{div}(\lambda \operatorname{grad} \theta)+p
$$

shows that with the hypothetic materials the time scale of the thermal transient, $\mathrm{t} / \mathrm{k}$, can be similar with the time scale of the electromagnetic transient. Thus, the time step of the thermal transient can be equal with the electromagnetic time step $60 \Delta \alpha /(2 \pi \mathrm{n})$. The value $\mathrm{k}=200$ of the time scale factor was considered by the numerical simulations.

\section{Results analysis}

The next results corresponds to the rotor speed $1000 \mathrm{rpm}$, angular step 3.6 degrees and the time step $\Delta \mathrm{t}=0.6 \mathrm{~ms}$. The transient variation of the temperatures starting from the ambient value in three points placed at the inner surface of the WP region, outer surface of the RING region and the interface between the WP and RING regions is presented in Fig. 19. 


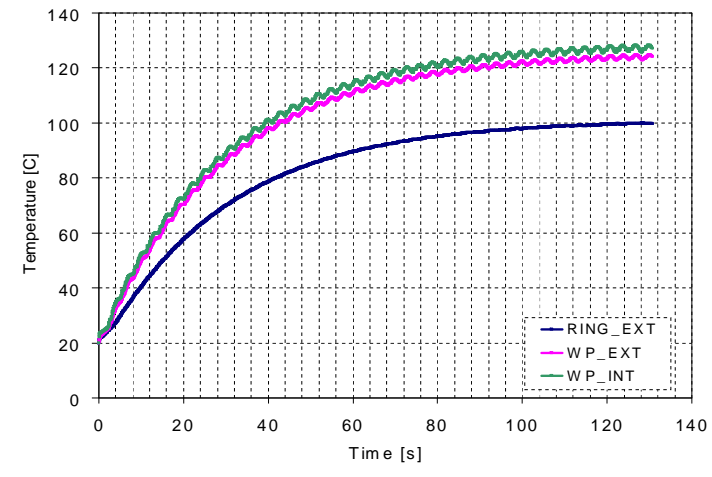

Fig. 19. Time dependence of temperature in three points on the stator

The chart of the eddy currents density at the time $20 \mathrm{~s}$ in the WP region is presented in Fig. 20.

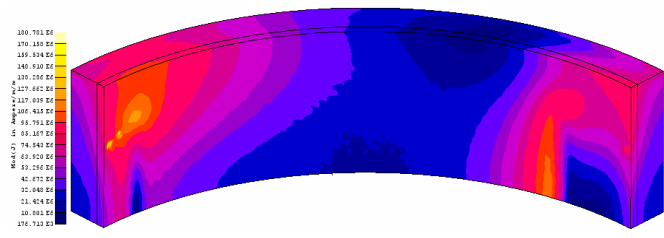

Fig. 20. Chart of the induced currents density WP region

In Fig. 21 a), b) are presented the time variations of the power induced in the WP and RING regions.

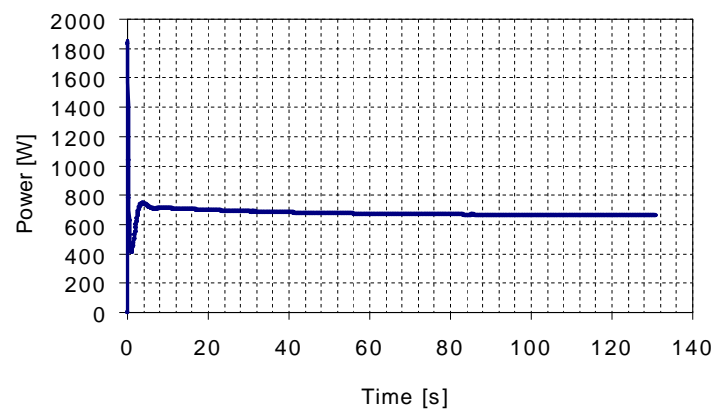

a)

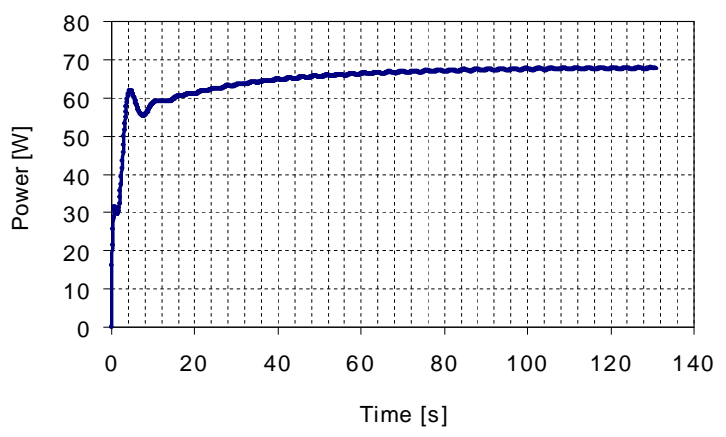

b)

Fig. 21. Time variation of induced power in WP and RING regions
The charts of the temperature on the AIR and WATER surface regions at the end of thermal transient are presented in Fig. 22 a), b).

Taking into account the steady state value $4876.7 \mathrm{~W}$ of the power induced in the stator of heater 2 and the increase of water temperature from the heater inlet to the outlet of $35{ }^{0} \mathrm{C}$, the flowrate of the heated water is 2 liter/minute.

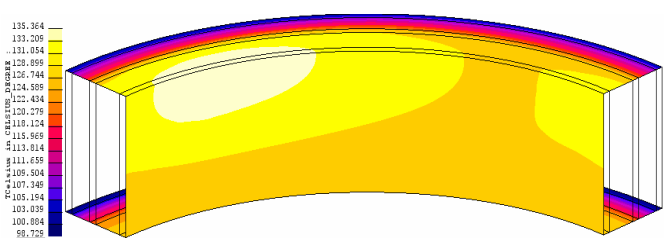

a)

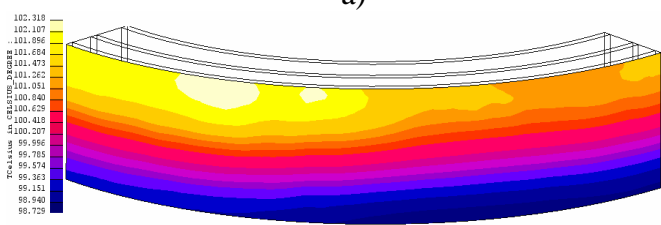

b)

Fig. 22. Temperature chart on AIR and WATER regions

\section{Conclusions}

The study of the eddy current heater based on finite element analysis in space domain and on the step-by-step in time domain analysis considers a simple strategy based on the transfer of steady state results at the end of electromagnetic transient to the transient heating problem and two complex strategies of the multiphysic coupling between electromagnetic and thermal transients.

The finite element models presented in this paper increase the performances of computer aided design and optimization in the eddy current heater development.

\section{References}

[1] V. Firețeanu, O. Nebi: "Finite Element Electromagnetic 2D Model of an Eddy Current Heater with Rotating Permanent Magnets" - $9^{\text {th }}$ International Conference on Applied and Theoretical Electricity ICATE 2008, Craiova, October 9-11, 2008.

[2] R. Araneo, A. Geri, M. Maccioni, G.M. Veca: "Eddy Current Induction Heating of a Conducting Cylinder in a Magnetic Field" in Proc. of International Symposium on Heating by Electromagnetic Sources, Padua, June 19-22, 2007.

[3] V. Firețeanu, T. Tudorache, M. Popa, S. Paşca: "Finite Element Analysis of Aluminum Billet Heating by Rotation in DC Magnetic Fields" in Proc. of XXIV UIE International Congress, Krakow, May 19-22, 2008.

[4] O. Nebi, V. Firețeanu: "Finite Element Analysis of a Permanent Magnets Heating Device" in $6^{\text {th }}$ International Symposium on Advanced Topics in Electrical Engeneering ATEE 2008, Bucharest, November 20-21, 2008. 